\title{
ELEVAGE LARVAIRE ET PRODUCTION DE JUVÉNILES ESTIVAUX D'OMBRE COMMUN (THYMALLUS THYMALLUS L.) AVEC UN NOURRISSAGE EXCLUSIF A L'ALIMENT SEC
}

\author{
H. CARMIE, L. JONARD
}

\author{
Délégation Régionale du Conseil Supérieur de la Pêche, \\ 84, avenue du Puy-de-Dôme - 63000 CLERMONT-FERRAND, FRANCE
}

\section{RÉSUME}

Jusqu'au début des années 80 , le démarrage des larves d'Ombre commun (Thymallus thymallus $L$.) n'est réputé possible qu'avec du zooplancton vivant ou congelé. Le développement du plancton au printemps dans des bassins de la Salmoniculture Expérimentale du Conseil supérieur de la Pèche (Augerolles, Puy-de-Dóme) est faible certaines années (climat rigoureux), malgré des amendements minéraux et organiques. La production moyenne de juvéniles d'ombre de 2 mois dans des bassins de 300 à $650 \mathrm{~m}^{2}$ varie de 4 à 8 ind./m² (survie inférieure à $30 \%$ ). En 1984, des expérimentations confirment l'efficacité d'un aliment sec expérimental (I.N.R.A.) à base de foie et de protéines unicellulaires, testé en 1983 sur un petit lot de larves. La survie après 4 semaines d'élevage est égale à $93 \%$, et le poids individuel moyen plus du double de celui noté avec de la farine pour saumon ou des nauplii d'artémies vivants ou congelés. Des essais réalisés en 1986 à l'échelle d'une pisciculture de production, avec un nourrissage exclusif à l'aliment sec, donnent de bons résultats d'élevage: survie et poids individuel moyen égaux respectivement à $93 \%$ et $0,9 \mathrm{~g}$ après 7 semaines de nourrissage, et à $91 \%$ et $4,5 \mathrm{~g}$ après 16 semaines. Une fiche technique présente les principales caractéristiques de l'élevage intensif des juvéniles d'Ombre commun.

\section{STARTING OF GRAYLING (THYMALLUS THYMALLUS L.) LARVAE AND PRODUCTION OF ESTIVAL JUVENILES EXCLUSIVELY USING DRY FOOD}

\section{ABSTRACT}

Until the beginning of eighties, starting of grayling (Thymallus thymallus L.) lavae was said possible only with live or frozen zooplancton. Some years the developement of plancton in ponds of the experimental hatcherie of Conseil Supérieur de la Pêche (Augerolles, Puy-de-Dôme) is low (rigorous climate) in spite of mineral and organic improvements. The mean production of two months old grayling juveniles in $300-650 \mathrm{~m}^{2}$ ponds ranges from 4 to 8 fishes $/ \mathrm{m}^{2}$ (survival lower than $30 \%$ ). In 1984, experiments confirme the efficiency of an experimental dry pelleted diet (I.N.R.A.) based on liver and single cell proteins, tested in 1983 with a little batch of larvae. After 4 weeks rearing the suvival was $93 \%$ and mean weight more than twice the value noted with salmon commercial meal, or live or frozen artemia nauplii. Trials realised in 1986 on a scale of a production plant, exclusively using dry food, give good rearing results : survival and mean weight equal to $93 \%$ and $0,9 \mathrm{~g}$ after 7 weeks teeding, $91 \%$ and $4,5 \mathrm{~g}$ after 15 weeks. A technical note presents the main characteristics of the intensive rearing of grayling juveniles.

\section{INTRODUCTION}

L'Ombre commun suscite un intérêt croissant de la part des pêcheurs, et en particulier de ceux opérant à la mouche, qui peuvent le capturer jusqu'au 31 décembre dans les eaux de deuxième catégorie.

Cet engouement s'accompagne d'une demande croissante en juvéniles en vue de soutenir les peuplements naturels, ou plus souvent d'implanter l'espèce sur des portions de cours d'eau où les caractéristiques morphodynamiques et la qualité de l'eau semblent favorables. Les essais d'introduction de l'espèce se sont souvent traduits par un échec. Les collectivités piscicoles pouvaient difficilement s'approvisionner en juvéniles à un stade autre que "alevin à la résorption de la 
vésicule", et leur coût élevé ( 3 à 4 fois supérieur, pour un mème stade, à celui de la Truite commune) ne les incitait pas à effectuer les opérations de repeuplement plusieurs années consécutives. La Salmoniculture Expérimentale du Conseil Supérieur de la Pèche à Augerolles (Puy-de-Dôme) a été le premier établissement à constituer un cheptel de géniteurs (CUINAT, 1978), puis à maîtriser la reproduction artificielle suite notamment à des études sur la maturation sexuelle (CARMIE et al., 1985; MAISSE et CARMIE, 1987). Certains pisciculteurs privés achetaient des œufs issus de géniteurs sauvages capturés au moment de la reproduction, généralement dans des rivières de Bavière ou de pays scandinaves. Le premier nourrissage des larves s'effectuait avec du plancton. des essais de démarrage avec de l'aliment sec ou divers régimes alimentaires s'étant soldés par un échec.

A la Salmoniculture d'Augerolles, l'élevage des alevins d'Ombre commun dans des bassins à plancton (apport d'engrais minéraux et organiques) donne généralement des résultats modestes (Tableau 1). Certaines années, la rigueur du climat au début du printemps freine le développement du plancton. La prédation par les larves de dytiques (dytiscidae) est importante, et la survie juqu'à l'áge de deux mois est inférieure à $30 \%$. Les aléas et sujétions liés à ce type d'élevage nous ont incité à étudier les possibilités de démarrage des larves en laboratoire. Le jaune d'œuf en poudre, la moule broyée, un aliment semi-humide, un aliment larvaire du commerce sont testés au début des années 80 sans succès : très faible croissance, survie après 24 jours d'élevage comprise entre 0 et $31 \%$ (MORELET, 1983 ; CARMIE et CUINAT, 1984). En 1983, le démarrage à Augerolles de 1.800 larves avec un aliment expérimental à base de levures, mis au point par le Laboratoire de Nutrition et d'Elevage des Poissons (I.N.R.A., St-Pée-sur-Nivelle) donne des résultats encourageants. Des régimes de composition voisine s'étaient révélés performants sur la Carpe (Cyprinus carpio) (DABROSKI et al., 1983) et le Corégone (Coregonus schinzi palea) (DABROSKI et al., 1984), dont le démarrage à l'aliment sec était, comme pour l'Ombre commun, réputé impossible. D'après DABROSKI (1983), les corégones développent un estomac à un stade plus avancé de leur ontogénèse comparativement à la plupart des salmonidés, qui possèdent des glandes gastriques dans l'estomac et des caeca pyloriques différenciès avant leur émergence et leur alimentation sur substrat exogène ; d'où la nécessité d'incorporer des protéines unicellulaires dans l'aliment sec composé lors du démarrage des larves. Le développement du tractus digestif chez les thymallidae est intermédiaire entre ceux des salmonidae et des coregoneidae (LUCZYNSKI et al., 1986).

Cet article décrit les deux principales étapes de la maîtrise de l'élevage intensif des juvéniles d'Ombre commun à la Salmoniculture d'Augerolles : essais comparatifs de l'efficacité de différents régimes alimentaires et structures d'élevage lors du démarrage en laboratoire (1984), obtention en 1986, en conditions expérimentales, de $\mathbf{5 0 . 0 0 0}$ estivaux avec nourissage exclusif à l'aliment sec.

Tableau I : Résultats d'élevage moyens obtenus en élevage semi-intensif dans des bassins (production de zooplancton) de 1981 à 1987.

Table I : Mean results of rearing grayling juveniles in ponds (zooplancton production) from 1981 to $1987.01=$ initial density $; 02=$ production $; P=$ mean individual weight.

\begin{tabular}{|c|c|c|c|c|c|}
\hline $\begin{array}{l}\text { STRATEGIE DE } \\
\text { PRODUCTION }\end{array}$ & MILIEU D'ELEVAGE & $\begin{array}{c}\text { DENSITE DE } \\
\text { DEPART : D1 }\left(\mathrm{N} / \mathrm{M}^{2}\right)\end{array}$ & $\begin{array}{l}\text { PRODUCTION : } \\
\text { D2 }\left(\mathrm{N} / \mathrm{M}^{2}\right)\end{array}$ & $\begin{array}{l}\text { POIDS INDIVIUUEL } \\
\text { MOYEN : } P \text { (G) }\end{array}$ & OBSERVATIONS \\
\hline \multirow{6}{*}{$\begin{array}{l}\text { PRODUCTION DE } \\
\text { JUVENILES DE } \\
8 \text { SEMINES }\end{array}$} & $\begin{array}{l}\text { BASSINS HEXAGO- } \\
\text { NAUX DE } 70 \mathbf{~ m}^{2}: \mathbf{A}\end{array}$ & $30 \wedge 45$ & $7 \times 20$ & $0.7 \times 1.6$ & P) $16 \mathrm{si} \quad 02<10$ \\
\hline & BASSINS EN IERRE & & $4 \lambda 8$ & $0.8 \times 1.3$ & \\
\hline & DE. $\begin{array}{c}300 \wedge 650 \mathrm{~m}^{2}: \\
\mathbf{B}\end{array}$ & $20 \wedge 30$ & 13 & 0.5 & UNE DONNEE \\
\hline & \multirow{3}{*}{$\begin{array}{l}\text { PF III ETANG DF } \\
2800 \mathrm{~m}^{2}: \mathbb{C}\end{array}$} & \multirow{3}{*}{$14.5 \wedge 16,5$} & 1,6 & $1.5 \times 1.9$ & TROIS ANNEES \\
\hline & & & 4,8 & 1.0 & UNE ANNEE \\
\hline & & & 6.7 & 1,1 & UNE ANNEE \\
\hline \multirow{3}{*}{$\begin{array}{l}\text { PRODUCTION DE } \\
\text { JUVENILES } \\
\text { D'UN ETE }\end{array}$} & & \multirow{2}{*}{$30 \times 45$} & $6 \times 12$ & $6.2 \times 8.6$ & AGE : 4 moIs \\
\hline & & & 34 & 2,8 & UNE DONMEE \\
\hline & B & $15 \times 20$ & 3 A 7 & $5.5 \times 7.2$ & AGE $: 5.5 \mathrm{mols}$ \\
\hline
\end{tabular}




\section{ESSAIS COMPARATIFS DE TECHNIQUES DE DÉMARRAGE INTENSIF EN LABORATOIRE (1984)}

\section{Matériel et méthodes}

Cinq régimes alimentaires sont testés lors du premier mois de nourrissage de juvéniles issus de géniteurs de $3^{\circ}$ génération en pisciculture (CARMIE et al., 1985) :

R1 : aliment I.N.R.A., composé de $50 \%$ de levure, $35 \%$ de foie de bœuf, $5 \%$ de mélange minéral, $5 \%$ d'huile de foie de morue, $5 \%$ de mélange vitaminique (BERGOT et al., 1986, régime $A$ ):

R2 : nauplii d'artémies (Artemia salina) congelés ;

R3 : aliment I.N.R.A. le matin, nauplii d'artémies congelés le soir;

R4 : nauplii d'artémies vivants;

R5 : farine démarrage commerciale pour saumon (60\% de protéines).

La nourriture est distribuée manuellement toutes les heures, de $8 \mathrm{~h}$ à $19 \mathrm{~h}$.

Trois structures d'élevage sont utilisées :

- des bassines cylindriques d'un volume utile de $50 \mathrm{l}$, disposant d'un débit de renouvellement d'eau de 2,5 $\mathrm{l} / \mathrm{mn}$ et d'une évacuation par trop plein. La densité, 40 ind./I au départ (7 mai) est ramenée à 25 ind./l après 15 jours d'élevage ;

- des cuvettes cylindro-coniques de $10 \mathrm{l}$, à évacuation centrale; débit $1 \mathrm{l} / \mathrm{mn}$; densité 85 ind./I ramenée à $\mathbf{4 0}$ ind./I :

- des clayettes de type californien ; volume utile : 40 litres ; débit : $5 \mathrm{l} / \mathrm{mn}$; densité: 60 ind./I ramenée à 40 ind./l.

L'efficacité des régimes $R 1$ à $R 3$ est étudiée dans les 3 structures d'élevage, celle des régimes R4 et R5 respectivement dans une cuvette cylindro-conique et une clayette.

Aucun traitement prophylactique ou curatif n'est effectué. La pesée hebdomadaire de $\mathbf{1 0 0}$ alevins par lot nous permet de calculer le taux de croissance instantané (RICKER, 1979):

Log w2-Log w1

$G=\frac{-12-t 1}{t 2}$, où w2 est le poids individuel moyen à la date t2 (en jours) et w1 celui à la date t1.

La mortalité est estimée à l'aide du taux quotidien de mortalité $(\mathrm{m})$

$m=1-\sqrt[t]{\frac{n+2}{n t 1}}$ où nt1 est le nombre initial

et nt2 le nombre au jour $\mathrm{t} 2 ; \mathrm{t}=\mathrm{t} 2-\mathrm{t} 1$

Pour une même période, ou une même structure d'élevage, une analyse de variance à 2 facteurs (aliment-structure d'élevage ou aliment-période d'élevage) nous permet de savoir si les différences observées entre les taux quotidiens de mortalité des différents lots d'autre part sont significatives. 


\section{Résultats}

\subsection{Croissance des juvéniles}

Dès la fin de la deuxième semaine d'expérimentation, et pour les trois milieux d'èlevage, le poids individuel moyen du lot nourri avec l'aliment I.N.R.A. (R1) est supérieur à celui du lot recevant des artémies congelées (R2).

Pour un même régime alimentaire le poids individuel moyen après un mois d'élevage dans la cuvette cylindro-conique est supérieur ou égal à celui observé dans le bac cylindrique ou la clayette.

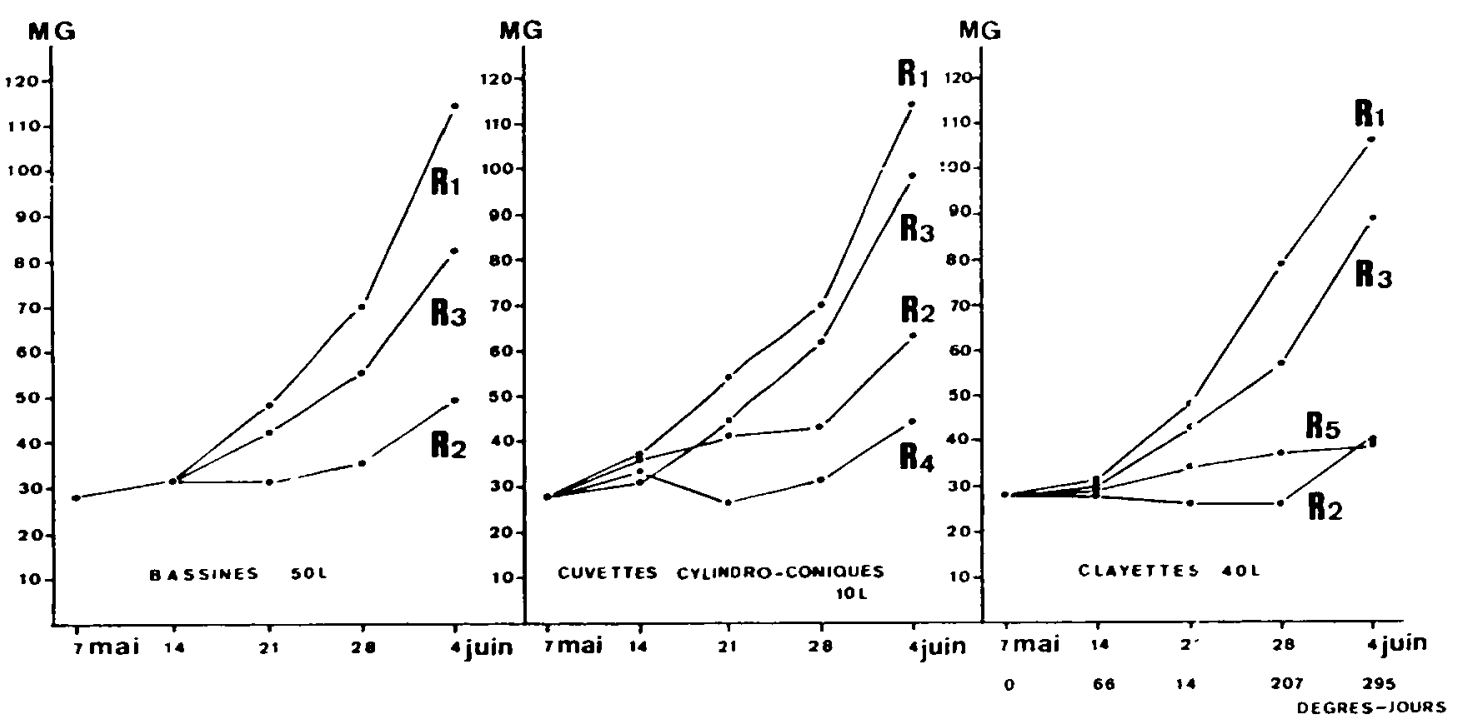

figure 1 : Evolution du poids individuel moyen pour les différents régimes alimentaires.

R1 : aliment I.N.R.A. ; R2 : artémies congelées ; R3 : R1 + R2; R4 : artémies vivantes ; R5 : aliment pour saumon.

figure 1 : Evolution of the individual fish weight for the different diets.

R1 : I.N.R.A. pelleted dry diet; R2 frozen artemla nauplii ; R3: H1 in the morning, R2 in the afternoon ; R4 : live artemla nauplii ; R5 : salmon dry food.

Lors de la deuxième puis de la troisième semaine d'élevage, le taux de croissance instantané noté avec le régime I.N.R.A. est significativement supérieur au taux du régime artémies congelées ( $\alpha=0,01$ et 0,05 respectivement).

Pour l'ensemble de la période d'élevage, le taux de croissance instantané est significativement supérieur avec le régime I.N.R.A. $(\alpha=0,05)$ comparativement aux régimes artémies vivantes (R4) (élevage en cuvette cylindro-conique) et farine commerciale (R5) (élevage en clayette).

Tableau II : Evolution du taux de croissance instantanée (\%) pour les différents régimes alimentaires.

Table II : Evolution of RICKER rate (G) for the different diets

$$
G=\frac{\log w 2-\log w 1}{t 2-t 1} \cdot 100
$$

$\mathbf{w 1}=$ individual body weight at date 11.

\begin{tabular}{|c|c|c|c|c|c|c|c|c|c|c|c|}
\hline & \multicolumn{3}{|c|}{ BASSINES } & \multicolumn{4}{|c|}{ CUVETTES CYLINDRO-CONIQUES } & \multicolumn{4}{|c|}{ CLAYETTES } \\
\hline & $\mathbf{R}_{\mathbf{1}}$ & $\mathbf{R}_{2}$ & $\mathbf{R}_{3}$ & $\mathbf{R}_{1}$ & $\mathbf{R}_{2}$ & $\mathbf{R}_{3}$ & $\mathbf{R}_{4}$ & $\mathbf{R}_{1}$ & $\mathbf{R}_{2}$ & $\mathbf{R}_{3}$ & $\mathbf{R}_{5}$ \\
\hline $07.05-14.05$ & 1.45 & 1,45 & 1,45 & 3,98 & 3.59 & 1,45 & 2.35 & 1.45 & 0 & 0.99 & 0.50 \\
\hline $14.05-21.05$ & 6.25 & 0 & 4.34 & 5,40 & 1.86 & 5.0 & -3.41 & 6.25 & -1.06 & 6.13 & 2.27 \\
\hline $21.05-28.05$ & 5,39 & 1.73 & 3.85 & 3,71 & 0.68 & 4.90 & 2.51 & 7.12 & 0 & 4.03 & 1.21 \\
\hline $28.05-4.06$ & 6.97 & 4,81 & 5.71 & 6,97 & 5.46 & 6.54 & 5.00 & 4.20 & 6.15 & 6.37 & 0.75 \\
\hline
\end{tabular}




\subsection{Survie}

Le taux de survie global au 6 juin est proche de $93 \%$ avec le régime I.N.R.A. compris entre $76 \%$ et $93 \%$ pour le régime artémies congelées, égal à $74 \%$ et $82 \%$ respectivement pour les régimes artémies vivantes et farine commerciale.

Pour chaque phase d'élevage (du 7 au 21.05 et du 21.05 au 6.06) dans les clayettes et les cuvettes, le taux quotidien de mortalité le plus faible est noté avec le régime I.N.R.A.. Pour une même structure d'élevage, sur l'ensemble de la période d'étude, la différence n'est pas significative $(\alpha=0,05)$.

Tableau III : Taux quotidiens de mortalité calculés pour chaque période ; survie globale.

Table III : Mortality dally rates (m) calculated for each period ; total survival (\%).

$$
m=1-\sqrt[t]{\frac{n t 2}{n t 1}} \quad \cdot 100 \quad \begin{aligned}
\text { withnt1 } & =\text { number at date } t 1 \\
n t 2 & =\text { number at date } t 2 \\
t & =t 2-t 1
\end{aligned}
$$

\begin{tabular}{|c|c|c|c|c|c|c|c|c|c|c|c|c|}
\hline & \multicolumn{3}{|c|}{ BASSINES } & \multicolumn{4}{|c|}{ CUVETIES CY INORO-CONIQUES } & \multicolumn{4}{|c|}{ CLAYETTES } \\
\hline & & $\mathbf{R}_{1}$ & $\mathbf{A}_{2}$ & $\mathbf{R} 3$ & $\mathbf{R}_{1}$ & $\mathbf{R}_{2}$ & $\mathbf{R}_{\mathbf{3}}$ & $\mathbf{R}_{4}$ & $\mathbf{R}_{\mathbf{1}}$ & $\mathbf{R}_{2}$ & $\mathbf{R}_{3}$ & $\mathbf{R}_{5}$ \\
\hline \multirow{4}{*}{$\begin{array}{c}7.05 \\
\mathrm{AU} \\
21.05\end{array}$} & $\begin{array}{c}\text { MOMBRE DE LARVES } \\
\text { AU.05 } \\
\text { W }\end{array}$ & 2000 & 2000 & 2000 & 850 & 850 & 850 & 850 & 2400 & 2400 & 2400 & 2400 \\
\hline & DERSITE ${ }^{(M / L)}$ & 40 & 40 & 40 & 85 & 85 & 85 & 85 & 60 & 60 & 60 & 60 \\
\hline & MORTALITES & 79 & 67 & 42 & 34 & 38 & 43 & 71 & 79 & 218 & 246 & 121 \\
\hline & $\begin{array}{c}\text { TAUX OUDTIDIEM } \\
\text { DE MORTKITE } \\
\text { m (\%) }\end{array}$ & 0.29 & 0.24 & 0.15 & 0.29 & 0.33 & 0.37 & 0.62 & 0.24 & 0.68 & 0.77 & 0.37 \\
\hline \multirow{4}{*}{$\begin{array}{c}21.05 \\
\text { AU } \\
6.06\end{array}$} & N AU 21.05 & 1250 & 1250 & 1250 & 400 & 400 & 400 & 400 & 1600 & 1600 & 1600 & 1600 \\
\hline & $n \quad(M r)$ & 25 & 25 & 25 & 40 & 40 & 40 & 40 & 40 & 40 & 40 & 40 \\
\hline & m & 43 & 144 & 54 & 11 & 12 & 16 & 76 & 47 & 262 & 177 & 217 \\
\hline & $\mathbf{m}(\boldsymbol{x})$ & 0.22 & 0.76 & 0.28 & 0.17 & 0.19 & 0.25 & 1.31 & 0.19 & 1.11 & 0.73 & 0.91 \\
\hline \multicolumn{2}{|c|}{$\begin{array}{l}\text { POURCENTACE DE SURVIE } \\
\text { DU } 7.05 \text { AU } 6.06\end{array}$} & 92.7 & 85.5 & $93: 6$ & 93.3 & 92.6 & 91.1 & 74.2 & 93.8 & 76.0 & 79.8 & 82.1 \\
\hline
\end{tabular}

\section{PRODUCTION D'OMBRETS DE 16 SEMANNES AVEC NOURRISSAGE EXCLUSIF A L'ALIMENT SEC (1986)}

Suite à ces essais comparatifs qui nous ont montré la supériorité de l'aliment I.N.R.A. et du bac cylindro-conique pour l'élevage des larves d'Ombre commun, nous avons mis en place une expérimentation dont le but était de définir les meilleures conditions d'élevage jusqu'au stade ombret de 16 semaines.

\section{Materiel et methodes (fig 2)}

L'élevage des larves débute le 12 et le 17 mai dans 4 poches en filet (vide de maille $1 \mathrm{~mm}$ ) de forme parallélépipédique fixées sur un cadre en bois et immergées dans des auges (volume utile $0,3 \mathrm{~m}^{3}$; débit de renouvellement d'eau filtrée : $12 \mathrm{~V} / \mathrm{mn}$ ). La densité de départ est de 100 à 130 ind./l. Tous les 2 jours, les poches sont transvasées dans des auges préalablement désinfectées. Elles sont éclairées individuellement $(60 \mathrm{~W})$.

Le 26 mai, les alevins de même âge sont regroupés. Six lots de 9.000 alevins (poids individuel moyen égal à $0,07 \mathrm{~g}$ pour les lots 1 et $2 ; 0,053 \mathrm{~g}$ pour les lots 3 à 6) sont mis en élevage dans des bacs cylindro-coniques (trémies), d'un volume utile de $1.5 \mathrm{~m}^{3}$, dotés d'un courant circulaire homogène (évacuation de fond centrale). Le débit de renouvellement d'eau, $10 \mathrm{Vmn}$, est doublé à la mi-juillet. 
Le 20 juin, 4.000 individus sont prélevés dans chaque lot et transvasés dans des bacs extérieurs de type suédois de $4 \mathrm{~m}^{2}$ (volume d'eau : $1,4 \mathrm{~m}^{3}$; débit : $20 \mathrm{Vmn}$, porté progressivement à $30 \mathrm{l} / \mathrm{mn}$ ). Le reliquat est conservé dans le bac conique d'origine (densité: 2,9 à 3,2 ind./I).

Les juvéniles sont nourris 12 heures par jour en continu (nourrisseurs automatiques) avec de l'aliment I.N.R.A. (taille $200-400 \mu$ la première semaine, $200-600 \mu$ la deuxième semaine), mélangé avec de l'aliment commercial pour saumon $(200-600 \mu, 56 \%$ de protéines) à partir du 26 mai (proportions 2/3,1/3). La part de cet aliment augmente régulièrement pour atteindre $100 \%$ le 4 juin.

B
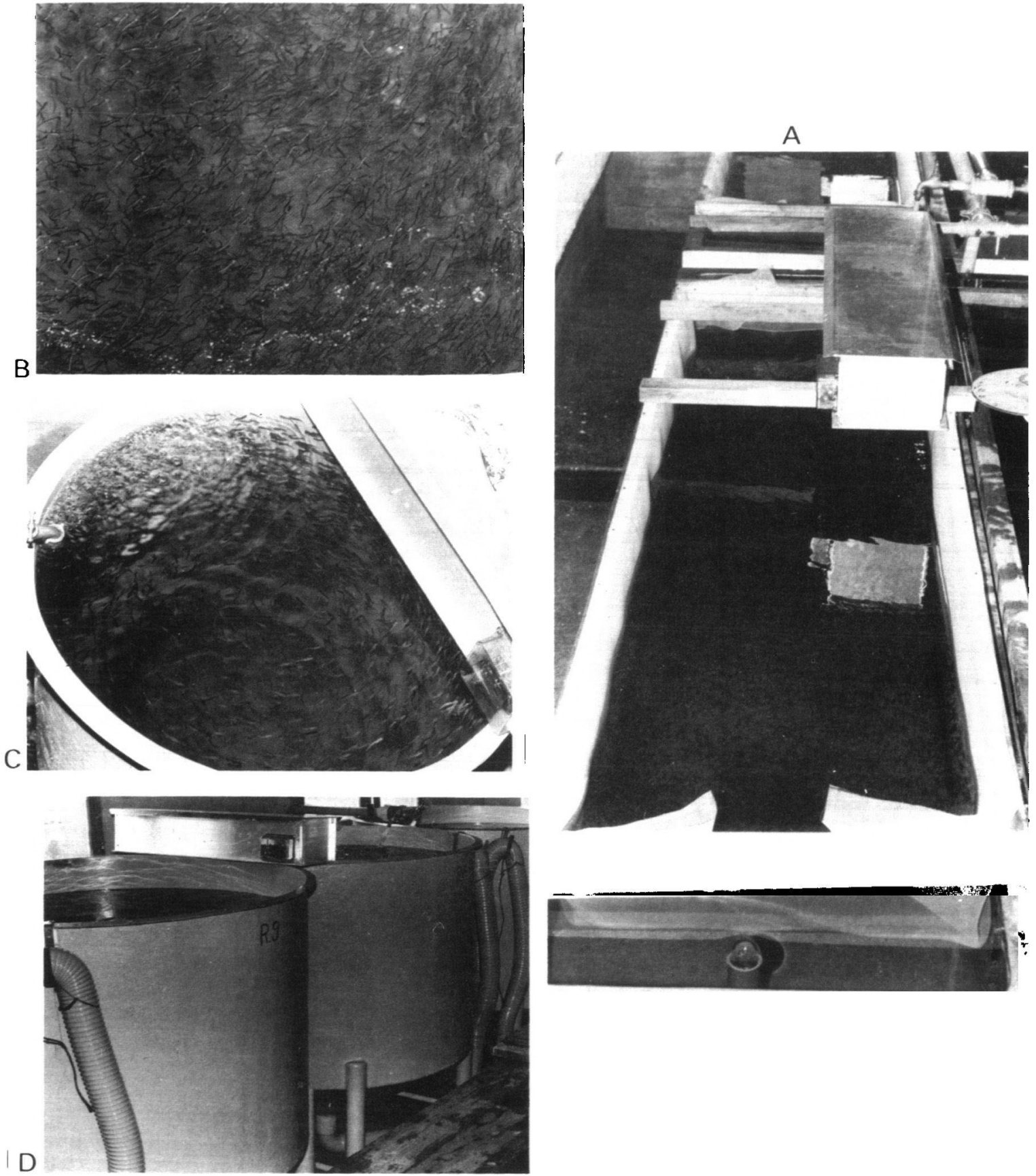

figure 2 : Démarrage des larves dans les nappes de filet (A, B). Elevage des juvéniles dans les bacs cylindro-coniques (C, D).

figure 2 : Starting of larvae in nets $(A, B)$.

Rearing of juveniles in cylindric tanks (C,D). 
Les températures moyennes journalières varient entre 13 et $16,5^{\circ} \mathrm{C}$ du 12 mai au 15 juin, entre 16,5 et $19^{\circ} \mathrm{C}$ du 16 juin au 30 août (maxima journaliers $\leqslant 20,5^{\circ} \mathrm{C}$ ).

La pesée de 200 individus par bac toutes les 3 à 5 semaines nous permet de calculer le taux de croissance instantané.

\section{Résultats}

\subsection{Crolssance (fig. 3)}

Du 26 mai au 19 juin, le taux de croissance instantané des lots 1 et $2(7,0$ et $7,3 \%)$ est légèrement inférieur à celui des lots 3 à $6(7,9$ a $8,1 \%)$ dont le poids individuel moyen de départ est supérieur $(0,07 \mathrm{~g})$.

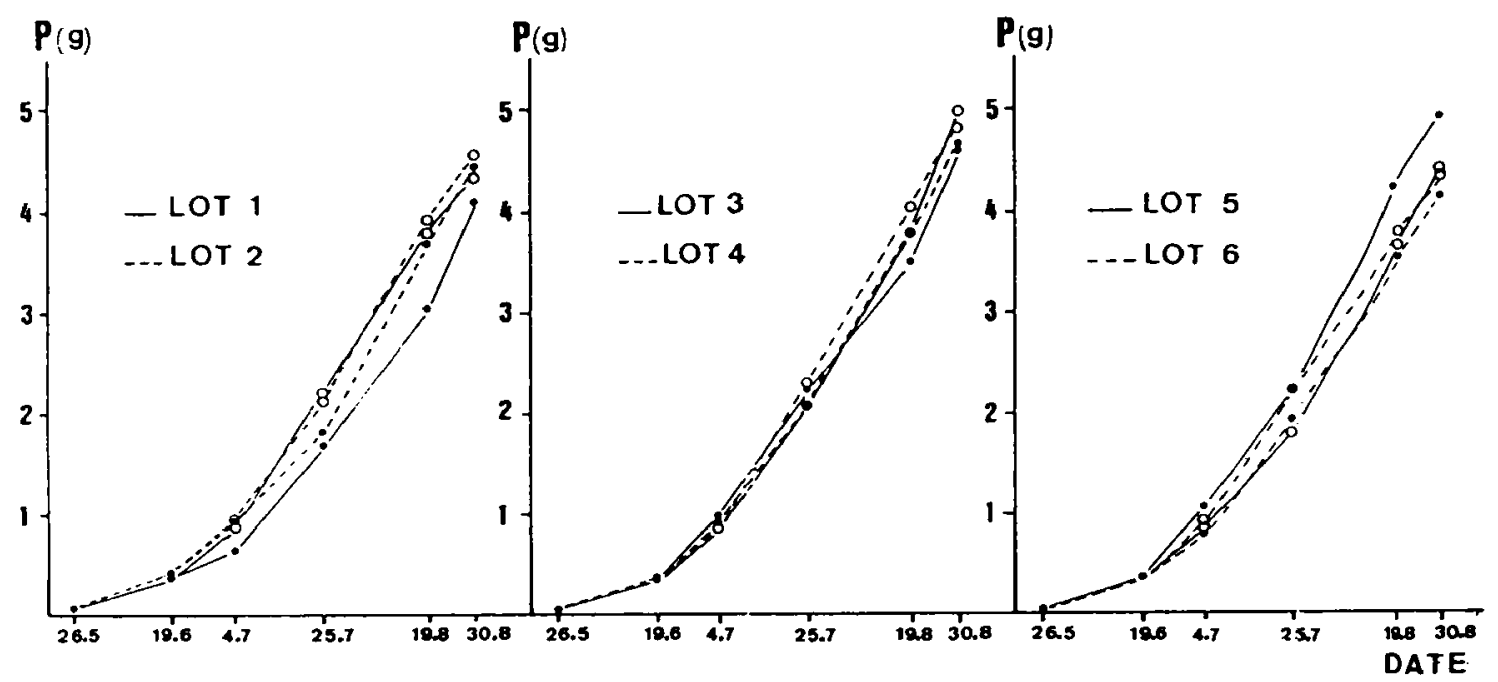

figure 3 : Evolution du poids individuel moyen dans chaque lot.
- bac cylindro-conique
- bac suédois

figure 3 : Evolution of the individual fish weight in each batch.

$$
\begin{aligned}
& \text { - cylindric tank } \\
& \text { o swedish tank }
\end{aligned}
$$

Pour la phase d'élevage estival (20 juin - 30 août), ainsi que pour chaque sous-période, le taux de croissance instantané dans les bacs suédois ne diffère pas significativement $(\propto \equiv 0,05)$ de celui observé dans les trémies. Sa valeur moyenne est de 5,7 et 5,8\% respectivement pour les trémies et les bacs suédois du 20 juin au 4 juillet; 3,9 et $4,1 \%$ du 4 au 25 juillet ; $2,4 \%$ du 25 juillet au 19 août; 1,9 et $1,6 \%$ du 19 au 30 août.

Au 30 août, le poids individuel moyen est similaire: $4,5 \mathrm{~g}$ pour les trémies, $4,6 \mathrm{~g}$ pour les bacs suédois. Pour le lot 5 la longueur totale moyenne est de 85,9 mm ( $\mathrm{n}=100, \quad \sigma=7,2 ; p=4,9 \mathrm{~g}$ ) (trémie) et $83,7 \mathrm{~mm}$ ( $n=100, \sigma=7,7 ; p=4,4 \mathrm{~g}$ ) (bac suédois).

\subsection{Survie}

La survie moyenne est de 98,1\% pour la phase premier nourrissage (12 au 26 mai) dans les poches de filet, 95,8\% pour la période 26 mai - 20 juin. Le taux quotidien de mortalité dans les bacs suédois est significativement supérieur $(\alpha=0,01)$ à celui des trémies pour la période 20 juin - 25 juillet; la survie est respectivement de 96,2 et 98,6\%. Dans les bacs suédois, la plupart des mortalités sont notées dans les 4 jours suivant le transvasement (21 ou 24 juin). Du 25 juillet au 31 août, la survie, tous lots et milieux d'élevage confondus, est égale à $99,4 \%$. Pour l'ensemble de la période d'élevage (12 mai au 31 août), on obtient une valeur de 91,0\%.

\section{DIscussion}

\section{Essais comparatifs de techniques de démarrage intensif en laboratoire (1984)}

Le poids individuel moyen après 15 jours de nourrissage avec l'aliment I.N.R.A., 0,05 g, est similaire à celui noté par LUCZYNSKI et al., (1986) avec le même aliment et des conditions d'élevage 
voisines. Après 4 semaines d'élevage, la valeur obtenue, $0,11 \mathrm{~g}$, est bien inférieure à celle indiquée par les auteurs polonais, $0,21 \mathrm{~g}$, et celle observée en 1986 après 26 jours d'élevage, $0,18 \mathrm{~g}$ (données non présentées). La croissance modeste durant la deuxième quinzaine s'explique certainement par une densité relativement élevée au début de cette phase d'élevage : 25 et 40 al./l. La survie pour l'ensemble de la période d'expérimentation, 93,7\%, est proche de celle observée en 1986 pour la même durée d'élevage - 94,9\% - et nettement supérieure à celle obtenue par LUCZYNSKI et al. (1986) : 53,3\%. Elle est à rapprocher de la valeur moyenne notée par BERGOT et al. (1986) avec des lots de corégones nourris avec le même aliment (régimes $A$ et $D$ ) : $96,4 \%$. Lors d'essais de nourrissage de corégones de la même espèce DABROSKI et al. (1984) observent après 35 jours d'alimentation une survie de 81 à $92 \%$ (selon la source de protéines) avec des aliments secs de composition voisine (57\% de protéines unicellulaires, $28 \%$ de foie de porc lyophilisé) et proche de $50 \%$ avec des nauplii d'artémies vivants.

La très faible croissance obtenue avec la farine pour saumon confirme l'inadaptation, pour le démarrage des larves d'Ombre commun, des aliments secs pour salmonidés actuellement dans le commerce.

\section{Production d'estivaux avec nourrissage exclusif à l'aliment sec (1986)}

La croissance régulière observée jusqu'à la fin août se poursuit au début de l'automne; malgré une température inférieure à $10^{\circ} \mathrm{C}$ après le 10 octobre, le poids individuel moyen de deux lots conservés à la pisciculture est doublé entre le 30 août et le 11 novembre (données non présentées). PERSAT (1976) note également sur la rivière Ain une croissance soutenue des ombrets de l'année pendant l'été et une bonne partie de l'automne alors que les sujets d'ảge 1 an+ présentent un ralentissement (voir un arrêt) de croissance pendant l'été, précédant une légère reprise avant l'hiver.

Au 31 août, le poids individuel de juvéniles, $8,4 \mathrm{~g}$, démarrés le 12 mai et élevés à partir du 26 mai dans une petite trémie de 300 litres (hors expérimentation car absence de replicat), est proche du double de celui noté dans les grandes trémies, alors que la densité d'élevage à partir du 20 juin est très voisine. Cela s'explique certainement par une meilleure disponibilité de l'aliment, et surtout un meilleur éclairage au fond du bac. DABROSKI (1983), s'appuyant sur les conclusions de travaux précédents, indique que de bonnes conditions de luminosité sont essentielles pour l'élevage des larves et des juvéniles de Coregonus pollan.

La croissance jusqu'à la fin juin dans les trémies est légèrement inférieure à celle observée dans les bassins en terre (nourriture naturelle); ce retard relatif est compensé pendant l'été.

L'excellente survie en début d'élevage est liée aux bonnes conditions sanitaires : transvasement des nappes de filet tous les deux jours dans des auges préalablement désinfectées, syphonage biquotidien de l'aliment non consommé; dans les trémies le courant circulaire et la position centrale de l'évacuation favorise l'autonettoyage. En 1983, lors des premiers essais de nourrissage avec l'aliment I.N.R.A., la survie après 3 semaines d'élevage dans des auges en ciment (fond rugueux) était proche de $50 \%$ (infection par des bactéries dont Aeromonas hydrophila). La valeur notée à la fin août, $91 \%$, est supérieure à celle obtenue en 1987 dans des conditions de production intensive: $85 \%$.

\section{CONCLUSIONS - PERSPECTIVES}

Les observations et recherches effectuées à la Salmoniculture Expérimentale du Conseil Supérieur de la Pêche à Augerolles ont permis de maîtriser la reproduction artificielle de l'Ombre commun puis l'élevage intensif des juvéniles (nourrissage exclusif à l'aliment sec). Les principales caractéristiques de cet élevage figurent à l'annexe $\mathrm{I}$.

Plusieurs centaines de milliers de larves ont été produites annuellement depuis la fin des années 70, dont 90.000 démarrés avec l'aliment I.N.R.A. en 1986, 200.000 en 1987, avec une survie jusqu'au stade ombret de quatre mois supérieure ou égale à $85 \%$. Elle n'aurait pas excédé $25 \%$ avec un démarrage dans des bassins à plancton, avec cependant, a priori, une meilleure faculté pour les sujets obtenus d'accomplir leur cycle vital après libération en milieu naturel, comparativement aux sujets nourris à l'aliment artificiel.

Les techniques d'élevage intensif ont été appliquées avec succés en 1988 aux piscicultures fédérales de la Dordogne et des Hautes-Pyrénées, sur des lots de 40.000 larves provenant d'Augerolles.

Le soutien des populations naturelles d'Ombre, ou l'implantation de l'espèce, nécessitent, au niveau de chaque bassin hydrographique concerné, la constitution d'un cheptel de reproducteurs de souche autochtone ou provenant de cours d'eau de caractéristiques voisines (régime thermique et qualité physico-chimique de l'eau, morphodynamique). Des ombres adultes ont été prélevés en septembre 1988 sur le haut bassin de l'Allier, dans une zone exempte de repeuplement, et stabulésá Augerolles. La reproduction artificielle de ces poissons, et leur "croisement" avec les géniteurs actuels, permettra d'obtenir des juvéniles de souche sauvage (première génération en pisciculture), et de souche "mixte". Les performances d'élevage, avec une alimentation artificielle et naturelle, de 
ces deux souches, et si possible leur faculté d'implantation en milieu naturel, seront comparées à celles de la souche "Augerolles", afin de définir les meilleures stratégies en matière de repeuplement en juvéniles d'Ombre commun.

\section{REMERCIEMENTS}

M. BERGOT et Nicole CHARLON, du Laboratoire de Nutrition et d'Elevage des Poissons, I.N.R.A. Saint-Pée-sur-Nivelle, nous ont aimablement fourni l'aliment sec expérimental.

M. TAVERNIER, stagiaire du C.R.E.U.F.O.P. de Montpellier et M. MESTAS, ouvrier piscicole mis à disposition par l'Union Régionale des Fédérations d'A.A.P.P. du Massif-Central, ont participé aux activités d'élevage et d'expérimentation. 


\section{ANNEXE 1 \\ FICHE TECHNIQUE SUR L'ÉLEVAGE INTENSIF DES JUVÉNILES D'OMBRE COMMUN (Nourrissage exclusif à l'aliment sec)}

Les observations réalisées et les résultats d'élevage obtenus de 1983 à 1987 dans un cadre expérimental ou de production de juvéniles de repeuplement nous permettent de dégager les principales caractéristiques de cet élevage.

\section{Le premier nourrissage}

II s'effectue 40 à 50 degrés-jours après la naissance, dans une poche de filet ou un bac à parois lisses, bien éclairé, à une densité de 100 à 120 ind./I.

L'aliment I.N.R.A., taille $200-400 \mu$ la première semaine (10 grammes par jour pour 10.000 alevins de $0,03 \mathrm{~g}), 200-600 \mu$ la semaine suivante, est distribué un minimum de 12 heures par jour en continu (nourrisseurs automatiques).

L'aliment non consommé est éliminé 2 fois par jour.

\section{Elevage des juvéniles}

Après 15 jours de nourrissage les juvéniles sont dépressés ou transvasés dans de nouveaux bacs. La forme cylindro-conique (trémie) permet de valoriser la surface disponible d'un bâtiment d'élevage.

L'aliment I.N.R.A., $400-800 \mu$ puis $600-1.200 \mu$, est progressivement remplacé par l'aliment commercial pour saumon $(200-600 \mu): 150 \mathrm{~g}$ par jour au total pour 10.000 alevins de $0,07 \mathrm{~g}$. Après un mois de nourrissage, l'aliment commercial $(200-800 \mu)$ est distribué seul.

Dix killogrammes d'aliment I.N.R.A. permettent d'obtenir environ 100.000 juvéniles âgés de 5 semaines (phase premier nourrissage comprise).

La densité d'élevage jusqu'à l'âge de 2 mois est de 6 ind./I dans des trémies de capacité moyenne $(1.500$ I). Pendant l'été cette densité est portée à 3 ind./l, valeur à pondérer d'après la température de l'eau et le taux de saturation en oxygène.

Les ombrets sont très sensibles au stress. II faut éviter les manipulations avec des températures de l'eau et (ou) de l'air élevées. Il est cependant utile de calibrer les sujets conservés en élevage à la fin de l'été.

\section{BIBLIOGRAPHIE}

BERGOT P., CHARLON N., DURANTE H., 1986. The effect of compound diets feeding on growth and survival of coregonid larvae. Arch. Hydrobiol. Beih., Ergebn., Limnol., 22, 265-272.

CARMIE H., CUINAT R., 1984. Elevage de l'Ombre commun. Premiers résultats obtenus à la Salmoniculture du Conseil Supérieur de la Pêche à Augerolles. Dactylographié, $19 \mathrm{p}$.

CARMIE H., MORELET B., MAISSE G., JONARD L., CUINAT R., 1985. Observations sur la reproduction artificielle de l'Ombre commun (Thymallus thymallus). Bull. Fr. Pêche Piscic., 296, 2-16.

CUINAT R., 1978. La Salmoniculture du Conseil Supérieur de la Pêche à Augerolles. Dactylographié, $15 \mathrm{p}$.

DABROWSKI K., 1983. Elevage des larves de corégones nourries à l'aliment sec et naturel. Synthèse bibliographique récente. Bull. Fr. Piscic., 291, 183-190.

DABROWSKI K., BARDEGA R., PRZEDWOJSKI R., 1983. Dry diet formulation study with common carp (Cyprinus carpio L.) larvae Z. Tierphysiol. Tierernähr. Futtermittelkd., 50, 40-52.

DABROWSKI K., CHARLON N., BERGOT P., KAUSHIK S., 1984. Rearing of coregonid (Coregonus schinzi palea Cuv. et Val.) larvae using dry and live food. I. Preliminary data - Aquaculture 41, 11-20.

LUCZYNSKI M., ZAPOROWSKI R.A., GOLONKA J.S., 1986. Rearing of European grayling, Thymallus thymallus $L$., larvae using dry and live food. Aquaculture and Fisheries Management, 17, 275-280.

MAISSE G., CARMIE H., 1987. Influence de la température sur l'ovulation de l'Ombre commun (Thymallus thymallus). Bull. Fr. Pêche Piscic., 305, 54-60.

MORELET B., 1983. Essai de maîtrise de la reproduction artificielle et de l'élevage de l'Ombre commun. Mémoire de fin d'études. Ecole Nationale Supérieure Agronomique de Rennes, $31 \mathrm{p}$.

PERSAT H., 1976. Principaux aspects de l'écologie de l'Ombre commun. Thèse de Doctorat de $3^{\circ}$ cycle de Sciences Biologiques, Université Claude Bernard Lyon I, 69 p. 